\title{
A Metabolic Pattern in Healthy Subjects Given a Single Dose of Metformin: A Metabolomics Approach
}

\begin{abstract}
Lina A. Dahabiyeh ${ }^{1 *}$, Muhammad Mujammami ${ }^{2,3}$, Tawfiq Arafat ${ }^{4}$, Hicham Benabdelkamel ${ }^{5}$, Assim A. Alfadda ${ }^{2,5}$ and Anas M. Abdel Rahman ${ }^{6,7,8 *}$

${ }^{1}$ Department of Pharmaceutical Sciences, School of Pharmacy, The University of Jordan, Amman, Jordan, ${ }^{2}$ Endocrinology and Diabetes Unit, Department of Medicine, College of Medicine, King Saud University, Riyadh, Saudi Arabia, ${ }^{3}$ University Diabetes Center, King Saud University Medical City, King Saud University, Riyadh, Saudi Arabia, ${ }^{4} J o r d a n$ Center for Pharmaceutical Research, Amman, Jordan, ${ }^{5}$ Proteomics Resource Unit, Obesity Research Center, College of Medicine, King Saud University, Riyadh, Saudi Arabia, ${ }^{6}$ Metabolomics Section, Department of Clinical Genomics, Center for Genome Medicine, King Faisal Specialist Hospital and Research Centre (KFSHRC), Riyadh, Saudi Arabia, ${ }^{7}$ Department of Biochemistry and Molecular Medicine, College of Medicine, Al Faisal University, Riyadh, Saudi Arabia, ${ }^{8}$ Department of Chemistry, Memorial University of Newfoundland, St. John's, NL, Canada
\end{abstract}

OPEN ACCESS

Edited by:

David E. Stec,

University of Mississippi Medical

Center, United States

Reviewed by:

Jean-Daniel Lalau,

University Hospital Center (CHU) of

Amiens, France

Xinwen Wang,

Northeast Ohio Medical University,

United States

*Correspondence:

Anas M. Abdel Rahman aabdelrahman46@kfshrc.edu.sa

Lina A. Dahabiyeh

I.dahabiyeh@ju.edu.jo

Specialty section:

This article was submitted to Drug Metabolism and Transport,

a section of the journal

Frontiers in Pharmacology

Received: 07 May 2021 Accepted: 28 June 2021

Published: 15 July 2021

Citation:

Dahabiyeh LA, Mujammami M, Arafat $T$, Benabdelkamel $H$, Alfadda $A A$ and Abdel Rahman AM (2021) A Metabolic Pattern in Healthy Subjects Given a Single Dose of Metformin: A

Metabolomics Approach.

Front. Pharmacol. 12:705932.

doi: 10.3389/fphar.2021.705932
Metformin is a widely prescribed medication for the treatment of type 2 diabetes mellitus (T2DM). It possesses effective roles in various disorders, including cancer, dyslipidemia, and obesity. However, the underlying mechanisms of metformin's multiple benefits are not fully understood. Herein, a mass spectrometry-based untargeted metabolomics approach was used to investigate the metabolic changes associated with the administration of a single dose of metformin in the plasma of 26 healthy subjects at five-time points; pre-dose, before the maximum concentration of metformin $\left(C_{\max }\right), C_{\max }$, after $C_{\max }$, and $36 \mathrm{~h}$ postdose. A total of 111 metabolites involved in various biochemical processes were perturbed, with branched-chain amino acid (BCAA) being the most significantly altered pathway. Additionally, the Pearson similarity test revealed that 63 metabolites showed a change in their levels dependent on metformin level. Out of these 63, the level of 36 metabolites was significantly altered by metformin. Significantly altered metformin-dependent metabolites, including hydroxymethyl uracil, propionic acid, glycerophospholipids, and eicosanoids, pointed to fundamental biochemical processes such as lipid network signaling, energy homeostasis, DNA lesion repair mechanisms, and gut microbiota functions that could be linked to the multiple beneficial roles of metformin. Thus, the distinctive metabolic pattern linked to metformin administration can be used as a metabolic signature to predict the potential effect and mechanism of actions of new chemical entities during drug development.

Keywords: metabolomics, metformin, branched-chain amino acids, mass spectrometry, glycerophospholipid, eicosanoids, type 2 diabetes mellitus, cancer

\section{INTRODUCTION}

Diabetes is a severe chronic disease affecting hundreds of millions of people worldwide, leading to high mortality and morbidity rates and increased health care costs (Guasch-Ferre et al., 2016). Metformin, dimethyl biguanide, is one of the most prescribed drugs for treating type 2 diabetes mellitus (T2DM) worldwide (Davies et al., 2018; Buse et al., 2020). It is an effective, safe, and 
relatively inexpensive anti-hyperglycemic agent associated with improved glycemic control and insulin sensitivity (Song, 2016). However, metformin does not alter glucose homeostasis in nondiabetic subjects (Sanchez-Rangel and Inzucchi, 2017). In addition, it has demonstrated cardioprotective effects that were not associated with clinical hypoglycemia or/and body weight gains (Sanchez-Rangel and Inzucchi, 2017; Foretz et al., 2019).

Metformin exerts its antidiabetic action mainly by acute suppression of hepatic gluconeogenesis through a transient inhibition of the mitochondrial respiratory chain complex I with a consequence activation of adenosine monophosphate (AMP)-activated protein kinase (AMPK) through liver kinase B1 (Viollet et al., 2012; Rena et al., 2017). Besides, metformin acts on multiple tissues and targets different pathways. It has been reported to reduce oxidative stress mainly by inhibiting the production (or neutralizing) of mitochondrial reactive oxygen species (ROS) (Bonnefont-Rousselot et al., 2003; Kane et al., 2010). Several disordered and complications are controlled and improved by metformin, including; metabolic and reproductive abnormalities of polycystic ovary syndrome (PCOS), cardiovascular complications associated with diabetes, cancer prognosis, and neurodegenerative diseases (Viollet et al., 2012; Rotermund et al., 2018; Foretz et al., 2019). Additionally, clinical studies have shown that metformin has beneficial effects on systemic inflammatory markers (Cameron et al., 2016) and weight loss in insulin-sensitive and insulin-resistant overweight and obese patients (Seifarth et al., 2013).

The pleiotropic properties of metformin and its numerous therapeutic areas suggest that various underlying mechanisms and metabolic pathways could be involved. Despite being introduced into the market for over 60 years, the mechanism of action of metformin remains partially explored and understood (Viollet et al., 2012; Foretz et al., 2014; Foretz et al., 2019). This urges the need for new and considerable efforts to understand better the cellular and molecular mechanisms of action of metformin.

Metabolomics is the comprehensive analysis of a set of small molecules (i.e., amino acids, lipids, and carbohydrates), referred to as metabolites within cells, biofluids, tissues, or organisms. It is a powerful analytical tool that is widely used to provide rich mechanistic information on drugs, and aid in identifying potential biomarkers that can be used to monitor the efficacy of drug therapies (Balashova et al., 2018; Jacob et al., 2019; Dahabiyeh et al., 2021). Pharmacometabolomics is an effective approach to capture the metabolic signatures linked to drug exposure and, therefore, improves the understanding of their underlying mechanisms of actions and allows individual differences recognition and drug toxicity prediction (Adam et al., 2016; Malkawi et al., 2018; Dahabiyeh et al., 2020). Several studies have reported the use of metabolomics to investigate the effect of metformin under pathological conditions, including T2DM (Bao et al., 2009; Adam et al., 2016), obesity (Zhu et al., 2013), cancer (Liu et al., 2016), metabolic syndrome (Ryan et al., 2020), and PCOS (Vinaixa et al., 2011). In this study, a single dose of metformin was given to healthy subjects. A label-free mass spectrometry-based untargeted metabolomics approach was used to identify metabolic dysregulation and pathways associated with metformin excreated levels (metformindependent metabolites). The dysregulated metformin-dependent metabolites could provide novel insights into the underlying biological pathways impacted by metformin administration.

\section{MATERIALS AND METHODS}

\section{Subject Recruitment and Study Design}

Subject recruitment and blood sample collection were conducted at Jordan Center for Pharmaceutical Research in Amman, Jordan. The Institutional Review Board reviewed and approved the study at Jordan Center for Pharmaceutical Research at Amman, Jordan (IRB-01-R02), and Institutional Review Board (IRB) at King Saud University (approval number E-19-4234) and written informed consent was obtained from all participants. Twenty-six healthy male subjects, aged 18-50 years, were enrolled in the study, whereas individuals with recorded health conditions were excluded. Each subject received a single oral dose of $500 \mathrm{mg}$ metformin hydrochloride film-coated tablet under standard fed conditions. A night before drug administration, all subjects were served standard dinner at least $10 \mathrm{~h}$ before metformin intake. Standard breakfast was served 30 min before drug administration, while standard lunch and dinner were served 6 and $12 \mathrm{~h}$ post drug administration. Individual serum concentrations of metformin were assayed at different time points following drug administration to determine $\mathrm{C}_{\max }$ (unpublished data). For each participant, blood samples were collected in heparinized tubes at multiple time points, and only five-time points were selected for this metabolomics study; pre-drug administration, $1.5 \mathrm{~h}$ before the maximum concentration of metformin in the serum $\left(\mathrm{C}_{\max }\right), \mathrm{C}_{\max }, 2 \mathrm{~h}$ after $\mathrm{C}_{\max }$, and $36 \mathrm{~h}$ post-drug administration, culminating a total of 130 samples. In this study, we selected the above five-time points to give a comprehensive measure of the metabolic pattern of metformin.

Screening (up to 14 days pre-dose administration) and followup (up to 7 days post-dose administration) examinations including comprehensive medical history, electrocardiogram (ECG), physical examination, vital signs (blood pressure and heart rate) measurements, blood hematology, and blood chemistry were performed for all participants. HbAlc, urinalysis, and serology tests (HBs Ag, HCV Ab, HIV I andII) were only performed during the screening examination. In addition, blood glucose levels were monitored at different time points. The demographic and clinical data of the participants are presented in Table 1.

\section{Sample Preparation and Metabolite Extraction}

A total of 130 serum samples were subjected to label-free untargeted metabolomics analysis using high-resolution liquid chromatography-mass spectrometry (LC-MS). Metabolites were extracted as previously described (Aleidi et al., 2021). Briefly, to $100 \mu \mathrm{L}$ serum sample, $300 \mu \mathrm{L}$ of methanol and $10 \mu \mathrm{L}$ of $2.8 \mathrm{mg} / \mathrm{ml}$ DL-o-chlorophenylalanine internal standard were added. After centrifugation for $15 \mathrm{~min}$, the supernatant was mixed with cold 
TABLE 1 | Clinical and demographic data of recruited subjects ( $n=26$ male) during screening and follow-up periods.

\begin{tabular}{|c|c|c|}
\hline \multirow{2}{*}{$\begin{array}{l}\text { Clinical } \\
\text { and demographic data }\end{array}$} & \multicolumn{2}{|c|}{ Mean \pm SD } \\
\hline & Screening & Follow-up \\
\hline Body mass index (BMl, kg/m²), (range) & $25 \pm 3.8,(19.2-29.3)$ & - \\
\hline Age (years) & $31 \pm 9.2$ & - \\
\hline Blood pressure (mm Hg) & $\leq 120 / 80$ & $\leq 120 / 80$ \\
\hline Heart rate (beat/minute) & $69.6 \pm 4$ & $71.9 \pm 6.5$ \\
\hline \multicolumn{3}{|l|}{ Biochemistry } \\
\hline Glucose (mg/dl) & $98.2 \pm 7.9$ & $91.5 \pm 9.7$ \\
\hline Urea (mg/dl) & $29.8 \pm 5.9$ & $31.3 \pm 6.7$ \\
\hline Creatinine (mg/dl) & $1.04 \pm 0.14$ & $1.08 \pm 0.11$ \\
\hline Sodium (mmole/L) & $143.2 \pm 2.7$ & $143 \pm 1.9$ \\
\hline Potassium (mmole/L) & $4.3 \pm 0.2$ & $4.2 \pm 0.17$ \\
\hline GOT (u/L) & $21.2 \pm 6.9$ & $26 \pm 17.0$ \\
\hline GPT (u/L) & $26.2 \pm 11.1$ & $30 \pm 34.0$ \\
\hline $\operatorname{ALK}^{\mathrm{a}}(\mathrm{u} / \mathrm{L})$ & $105 \pm 19.0$ & $86 \pm 16.0$ \\
\hline Total protein (g/dl) & $7.4 \pm 0.5$ & $7.7 \pm 0.5$ \\
\hline Total bilirubin (mg/dl) & $0.47 \pm 0.14$ & $0.5 \pm 0.5$ \\
\hline $\mathrm{HbA1c}(\%)$ & $5.2 \pm 0.23$ & - \\
\hline \multicolumn{3}{|l|}{ Hematology } \\
\hline Hemoglobin (g/dl) & $16.1 \pm 1.0$ & $15.8 \pm 1.1$ \\
\hline Hematocrit (\%) & $47.7 \pm 2.9$ & $45.6 \pm 2.8$ \\
\hline R.B.C (1012/L) & $5.4 \pm 0.4$ & $5.3 \pm 0.3$ \\
\hline M.C.V (fL) & $88.2 \pm 4.3$ & $86.6 \pm 3.5$ \\
\hline M.C.H (pg) & $29.6 \pm 1.8$ & $30.0 \pm 1.6$ \\
\hline M.CH.C (g/dl) & $33.6 \pm 0.6$ & $34.6 \pm 0.7$ \\
\hline \multicolumn{3}{|l|}{ Differential Leucocytes Count } \\
\hline Leucocytes $\left(10^{9} / \mathrm{L}\right)$ & $7.8 \pm 2.0$ & \\
\hline Neutrophils (\%) & $62.3 \pm 3.7$ & $64.2 \pm 2.5$ \\
\hline Lymphocytes (\%) & $33.5 \pm 3.2$ & $32.0 \pm 2.5$ \\
\hline Monocytes (\%) & $3.4 \pm 1.1$ & $3.0 \pm 0.7$ \\
\hline Eosinophils (\%) & $0.73 \pm 0.7$ & $0.77 \pm 0.8$ \\
\hline Basophils (\%) & $0.04 \pm 0.2$ & $0.12 \pm 0.3$ \\
\hline Platelets $\left(10^{9} / \mathrm{L}\right)$ & $245.1 \pm 40.5$ & $272.1 \pm 46.2$ \\
\hline
\end{tabular}

Data are presented as mean \pm standard deviation.

GOT: glutamic oxaloacetic transaminase, GPT: glutamic pyruvic transaminase, ALK: Alkaline phosphatase, R.B.C: red blood cell, M.C.V mean corpuscular volume, M.C.H mean corpuscular hemoglobin, M.CH.C: mean corpuscular hemoglobin concentration. All lab tests were within the normal range with no significant difference between screening and follow-up periods with the exception of ALK

a Lab test values were significantly different between screening and follow-up periods (independent $\mathrm{t}$-test, $\mathrm{p}$ value $\leq 0.05$ )

acetonitrile $(\mathrm{ACN})$ and centrifuged again for $5 \mathrm{~min}$. Finally, the supernatant was dried, and before LC-MS analysis, the dried residue was reconstituted in 1:1 (v/v) methanol/water.

\section{Label-free Liquid Chromatography-Mass Spectrometry Untargeted Metabolomics Analysis}

Metabolomics profiling was performed by Ultimate 3000 LC combined with Q Exactive MS (Thermo Fisher Scientific, CA, United States) as reported in our recent publication (Aleidi et al., 2021). Briefly, extracted metabolites were first separated using an ACQUITY UPLC HSS T3 $(100 \times 2.1 \mathrm{~mm} 1.8 \mu \mathrm{m})$ and a mobile phase composed of solvent A $(0.05 \%$ formic acid-water $)$ and solvent $\mathrm{B}(\mathrm{ACN})$ with a gradient elution over $16 \mathrm{~min}$ applied at $300 \mu \mathrm{l} / \mathrm{min}$ flow rate. MS spectra were acquired in full MS scan in the range $m / z 50-1,500$, with 25,000 enhanced mass resolution and a frequency 15 spectra per second. The capillary voltage was
3000 and $3200 \mathrm{~V}$ for positive and negative ionization modes, respectively. The fragmentation was achieved for MSMS experiments at $175 \mathrm{~V}$, with nebulizer gas at $35 \mathrm{bsi}$, and gas temperature $450^{\circ} \mathrm{C}$. Chromatographic and MS parameters (under positive and negative ionization modes) were kept as reported previously (Aleidi et al., 2021). Pooled samples prepared the quality control (QC) sample. A QC injection was performed every 10 LC-MS sample runs. In total, there were 18 QC samples injected and analyzed.

\section{Metabolites Identification}

The raw data were acquired and aligned using the compound discoverer software (Thermo Fisher Scientific, United States) based on the $m / z$ value and the ion signals' retention time. Then, the chemical structures of metabolites were identified by matching the data obtained from accurate mass analysis and MS/ MS fragmentation with data available in the online databases; the Human Metabolome Database (www.hmdb.ca), METLIN (www. metlin.scripps.edu), and the Mass Bank (www.massbank.jp).

\section{Statistical Analysis}

Multivariate statistical analysis was performed using SIMCAP+14 from Umetrics $\mathrm{AB}$ (Umeå, Sweden). For the identified metabolites, peak intensities at each time point were normalized, Parito-scaled, and log-transformed. The processed peak intensities were used to generate principal component analysis (PCA) and orthogonal partial least squaresdiscriminant analysis (OPLS-DA) models to overviewing the metabolic differences between the various time points. The robustness of the created models was evaluated by the fitness of model $\left(\mathrm{R}^{2} \mathrm{Y}\right)$ and predictive ability $\left(\mathrm{Q}^{2}\right)$ values (Worley and Powers, 2013). MetaboAnalyst version 4.0 (McGill University, Montreal, Canada) (http://www.metaboanalyst.ca) was used to visualize the affected metabolic pathways as of metformin intake (Chong et al., 2018).

Univariate analysis using one-way analysis of variance (ANOVA) and post-hoc Tukey's analysis method was performed for time points. Significantly differentially expressed metabolites were determined based on false discovery rate (FDR) adjusted $p$-value less than 0.05 and fold change (FC > 1.5, <0.67). The total sample median was used to normalize the signal, ensure normal distribution, and represent Z-score. The metabolic patterns connected to metformin's action were developed using Venn diagrams and Pearson similarity test (MPP Software, Agilent Inc., CA) as discussed before (Gu et al., 2020).

\section{RESULTS}

\section{Clinical and Demographic Data of Study Subjects}

Table 1 summarizes the demographic and clinical data of the 26 male subjects included in this study. All participants were healthy non-diabetic men. Screening and follow-up examinations revealed that all subjects had normal heart rates, blood pressure $(\leq 120 / 80 \mathrm{~mm} \mathrm{Hg})$, blood glucose, and HbAlc levels. Hematology and biochemistry tests were within the normal range 
A

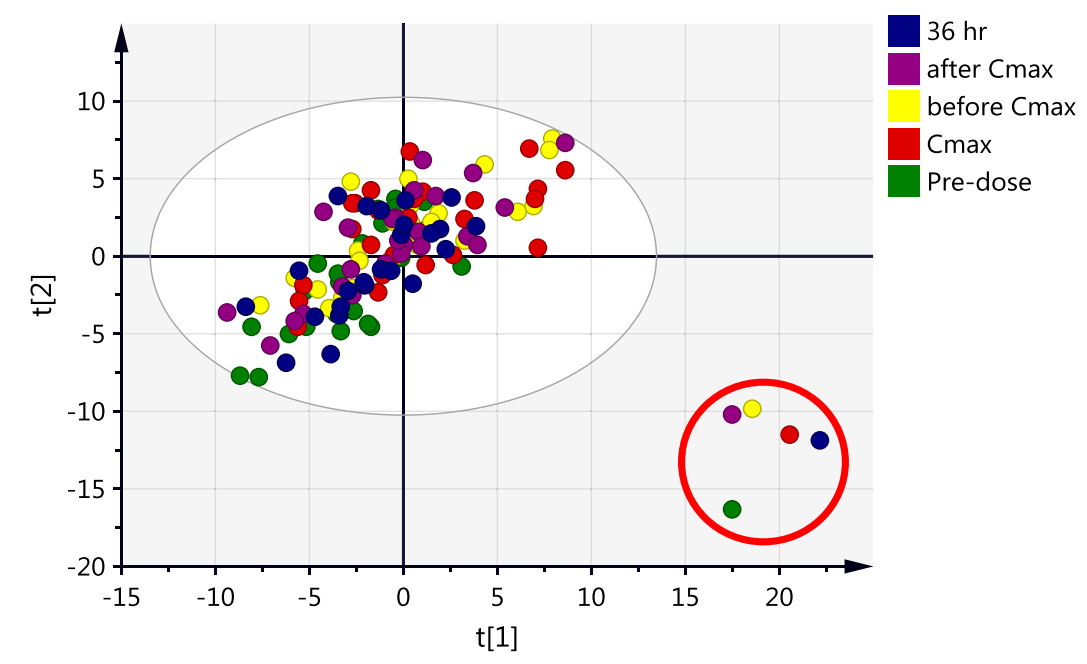

B

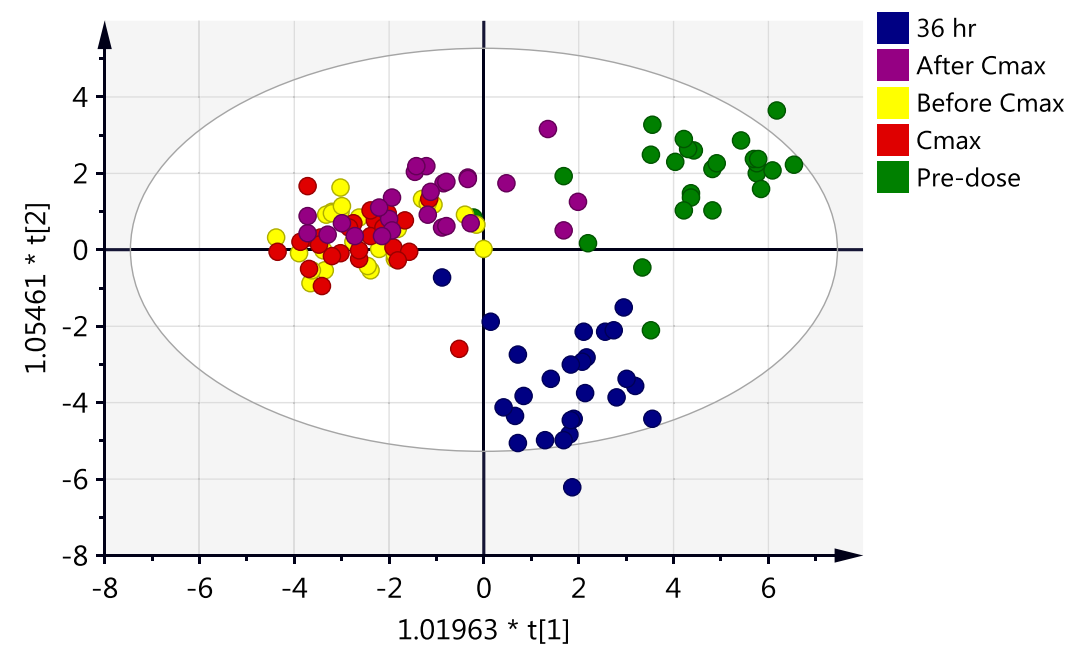

FIGURE 1 | Scores plots of the metabolite profile of serum samples obtained from 26 healthy subjects after single dose of metformin at five time points; pre-dose (baseline level, green), and 1.5 h before $C_{\max }$ (yellow), $C_{\max }$ (red), 2 h after $C_{\max }$ (purple) and 36 h post-drug administration (blue). (A) PCA ( $\left.R_{2} X=0.71, Q^{2}=0.44\right),(B)$ OPLS-DA (R2X = 0.28, R2Y =0.40, Q2 = 0.27).

for all participants during screening and follow-up examinations with no significant difference between the two periods except for the alkaline phosphatase test (ALK known as ALP), Table 1. The level of the ALK enzyme was significantly lower in the follow-up period $(105 \pm 19)$ compared to the screening period $(86 \pm 16)$, which indicates that metformin can affect the circulating levels of ALK. Of note, one subject showed a substantial increase in the levels of the two liver enzymes, glutamic oxaloacetic transaminase (GOT, known as AST) and glutamic pyruvic transaminase (GPT, known as ALT), during the follow-up period compared to screening tests $(15,19$ in screening to 104,187 in follow up, respectively). Moreover, the same subject was the only one to show an increase in ALK level compared to others who showed decreased ALK levels during the follow-up period.

\section{Metabolites Detection and Multivariate Analysis}

A total of 4,456 and 3,182 mass ion features were detected in positive and negative ionization modes, respectively. Using the data of accurate masses and MS/MS fragments, 444 and 400 metabolites were putatively identified, in positive and negative ionization modes, respectively, with 110 metabolites commonly identified in both ionization modes. Metabolites identified by the two ionization modes were merged (734 metabolites) and exported for multivariate analysis using PCA and OPLS-DA (Figure 1). The data were deposited in MetaboLight (accession Number MTBLS2949.

Individual serum concentrations of metformin were assayed at different time points following drug administration to determine 
A

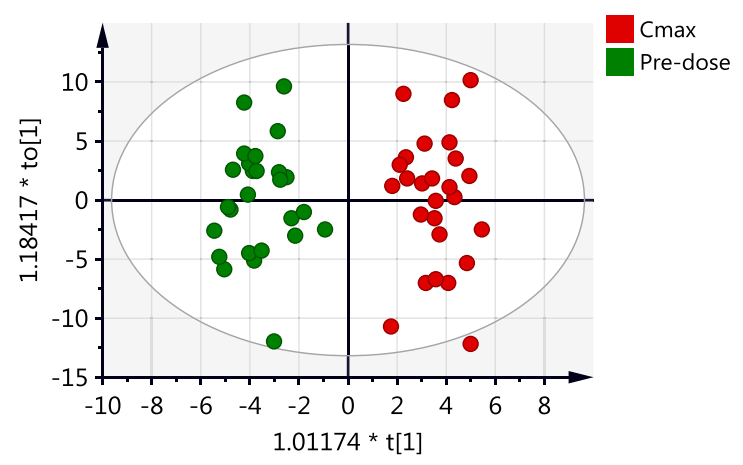

C

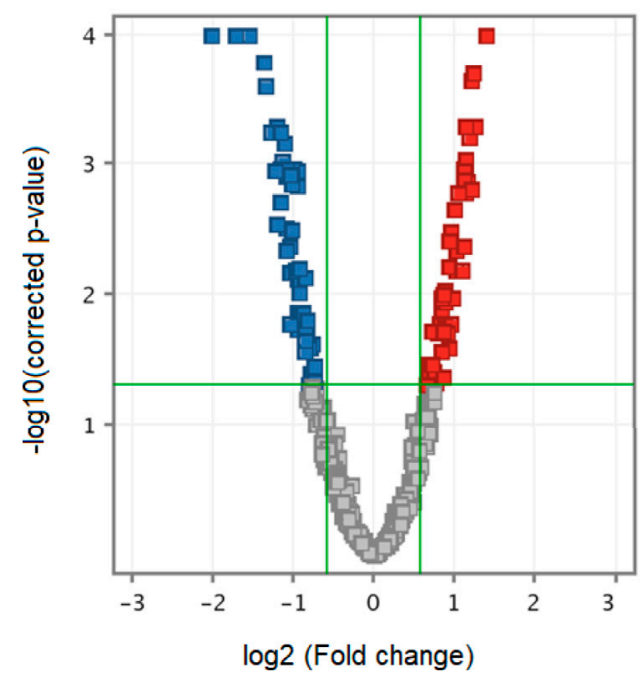

B

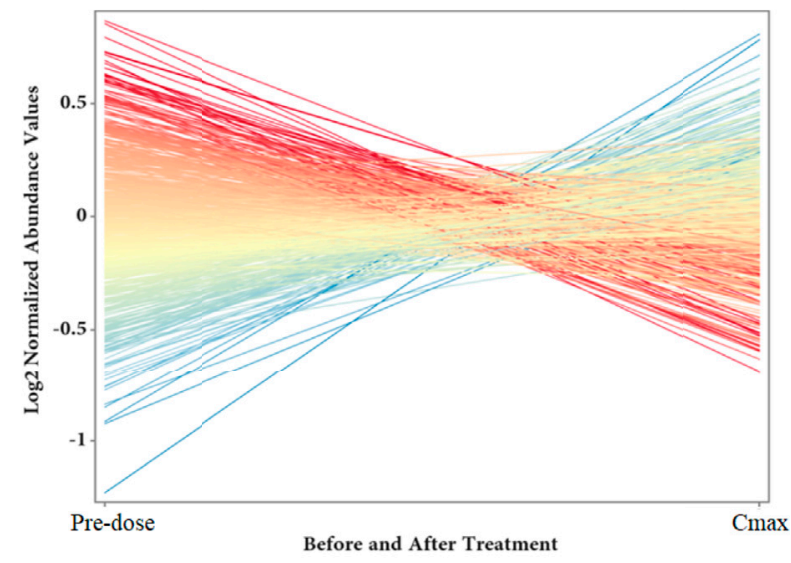

D

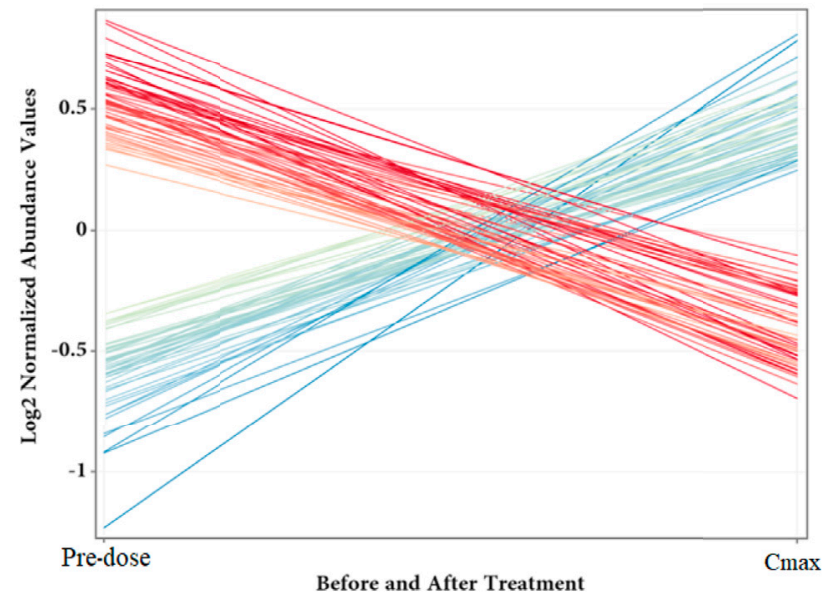

FIGURE 2 | Differentially expressed metabolites as of metformin administration based on a binary comparison between baseline level (pre-dose) and $\mathrm{C}_{\text {max }}$ level. (A) OPLS-DA (R2X = 0.26, R2Y = 0.92, Q2 = 0.62) scores plot of the metabolic profile of serum samples obtained from 26 subjects pre-dose (green) and at $C_{\text {max }}$ (red). (B) Out of the 734 identified metabolites, 111 were significantly dysregulated (presented as red and blue lines) while the remaining were unchanged (yellow lines). (C) Volcano plot of up (red, $n=57$ ) and down (blue, $n=54$ ) regulated metabolites. (D) The differentially altered metabolites as of metformin administration. Blue and red refer to up-and down-regulated metabolites, respectively.

$\mathrm{C}_{\max }$ (unpublished data). Five-time points reflecting different metformin levels in the circulation were selected; pre-dose and four post-drug administration time points (before $\mathrm{C}_{\max }, \mathrm{C}_{\max }$, after $\mathrm{C}_{\max }$, and $36 \mathrm{~h}$ ) for endogenous metabolomics association. Multivariate analysis (Figure 1) was performed to investigate group clustering and separation and identify potential outliers among the compared data time points. The PCA score plot (Figure 1A) overlaps with no separation or clustering achieved between the various time points. However, the PCA model clearly identified outliers that belong to the metabolic data of one subject at the five-time points (Figure 1A). The same subject had an increased level of liver enzymes during the follow-up period. The different metabolic patterns of this subject might be independent of metformin administration as all time-points, including pre- dose, are clustered outside the PCA model's confidence interval. Therefore, data for this participant were excluded from the dataset before further analyses.

The OPLS-DA model could not entirely separate the dataset obtained at different time points, and only partial separation could be noticed mainly due to the complexity of the dataset (Figure 1B). demonstrates that pre-dose and $36 \mathrm{~h}$ post-dose samples exhibited evident separation from the other time points. In contrast, the remaining three-time points (before $\mathrm{C}_{\max }, \mathrm{C}_{\max }$, and after $\mathrm{C}_{\max }$ ) were overlapped and did not show any separation or clustering. The previous findings indicate that the metabolic profile after $36 \mathrm{~h}$ of metformin administration did not retain a level close to the baseline. However, the circulating level of metformin could not be detected at $36 \mathrm{~h}$ post-administration (data not shown). Therefore, it is plausible 


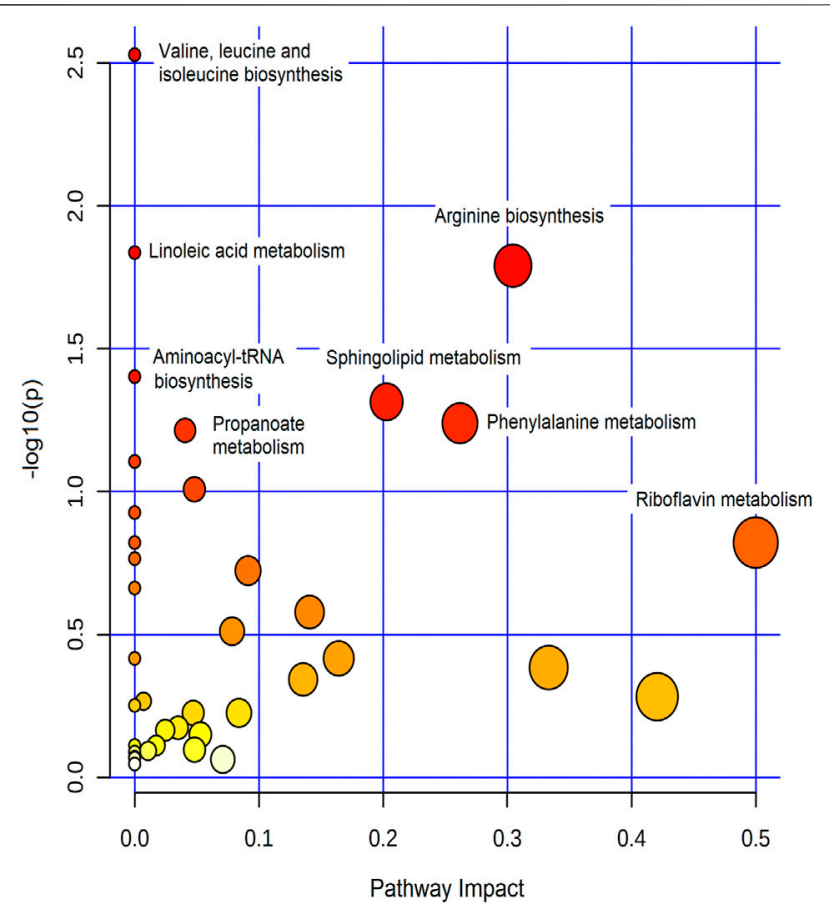

FIGURE 3 | Summary of the most affected pathways. The node color and size are based on the $p$-value and the pathway impact value, respectively.

that a longer time is needed after the intake of metformin for the metabolite level to return to a normal level even if the level of metformin in the circulation is very low.

\section{The Effect of Metformin on Circulating Metabolites in Healthy Subjects}

The effect of metformin on biological metabolites was examined by investigating its influence on the levels of the 734 putatively identified metabolites using mainly two-time points; pre-dose level (before treatment) and $\mathrm{C}_{\max }$ (post-drug administration associated with the highest level of metformin), as presented in Figure 2. OPLS-DA model showed evident separation between the two groups (Figure 2A), indicating that metformin administration has significantly altered the dynamic of different metabolic processes. Binary comparison using volcano plot (cutoff 1.5) revealed that out of 734 metabolites, 111 were dysregulated, of which 57 and 54 metabolites were up-and down-regulated as of metformin administration, respectively (Figures 2C,D). The identity of these metabolites is presented in Supplemenatry Table S1 in the supplementary material. The 111 altered metabolites are involved in various biological processes, including valine, leucine, and isoleucine biosynthesis, linoleic metabolism, arginine biosynthesis, and aminolylacyl-tRNA biosynthesis, as shown in Figure 3.

\section{Endogenous Metabolites Linked to Metformin Level}

Metabolites dysregulated endogenously, and their levels associated with metformin's level (Metformin-dependent metabolites) were identified by comparing the levels of differentially altered metabolites to metformin patterns at the five-time points. The Pearson similarity test $(\mathrm{R}=0.95-1)$ revealed that 21 metabolites (excluding metformin) showed a similar expression trend to metformin Figure 4A. Out of these 21 metabolites, 8 (excluding metformin) significantly increased their levels based on the binary comparison between baseline and $\mathrm{C}_{\max }$ levels (Figure 4B). These include 5-aminopentanoic acid, propionic acid, hydroxymethyl uracil, and ethyl phenyl sulphate (Figure 4C). On the other hand, 42 metabolites acted in an opposed manner to metformin levels, with 28 metabolites showing a significant decrease in $\mathrm{C}_{\max }$ level compared to baseline. Metabolites that opposed levels to metformin patterns mainly involved arachidonic and linoleic acid metabolisms and included glycerophospholipids(such as lysophosphatidic acid and lysophospholipid) and eicosanoids (prostaglandin H1, 11dehydrothromboxane B2), Figure 4C. Heatmap of the 36 metformin-dependent significantly perturbed metabolites and their levels at the five-time points is shown in Figure 4C.

Noteworthy, specific metabolites showed an interesting change in their levels compared to the metformin pattern (Figure 5). After metformin administration, the level of 6 and 12 metabolites was initially increased and decreased, respectively, followed by a subsequent linear decrease or increase, until returning to levels similar to pre-dose at $36 \mathrm{~h}$ post drug administration as shown in Figures 5A,B, respectively. Among these metabolites are several acylcarnitines and coumaric acid.

\section{DISCUSSION}

Metformin is a widely used biguanide drug due to its outstanding safety profile, low cost, and promising effects in T2DM, cancer, PCOS, weight reduction, and many other medical conditions. It exerts multiple effects through different signaling pathways. Extensive literature has investigated the role of metformin in various disorders. However, the underlying mechanisms of its multiple benefits remain to be elucidated. Moreover, examining the effect of metformin under pathological conditions makes identifying metabolites specifically altered due to metformin rather than the disease state difficult, particularly that many patients will take metformin in combination with other medications, which will definitely affect the metabolomics data. To the best of our knowledge, this is the first study to examine the effect of a single dose of metformin on the metabolic pattern of healthy subjects at different time points.

Metformin induced significant changes in several biochemical pathways, including amino acids and aminoacyl-tRNA biosyntheses, and fatty acid metabolism. Amongst them, alteration of branched-chain amino acids, BCAA (valine, leucine, and isoleucine), was the most significant pathway (Figure 3). BCAA are crucial regulators of energy homeostasis, glucose and lipid metabolism, gut health, and immunity (Nie et al., 2018). In addition, they serve as substrates to synthesize nitrogenous compounds and play a critical role in protein and fatty acids syntheses (Ma et al., 2017; Nie et al., 2018). Therefore, metabolic imbalance in BCAA levels (increased catabolic flux or 
A

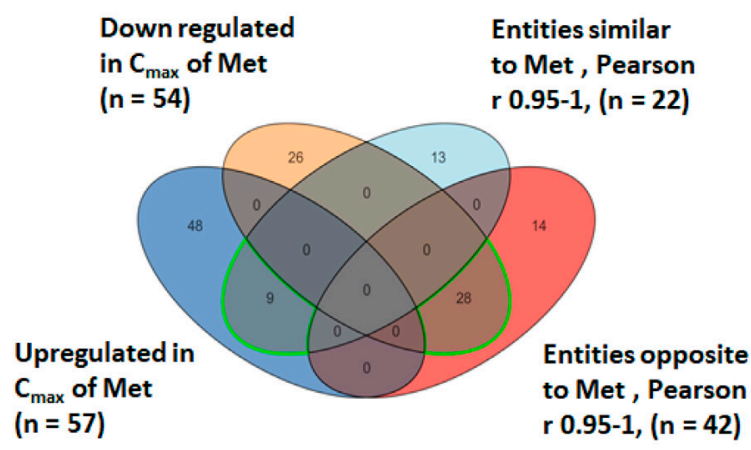

B

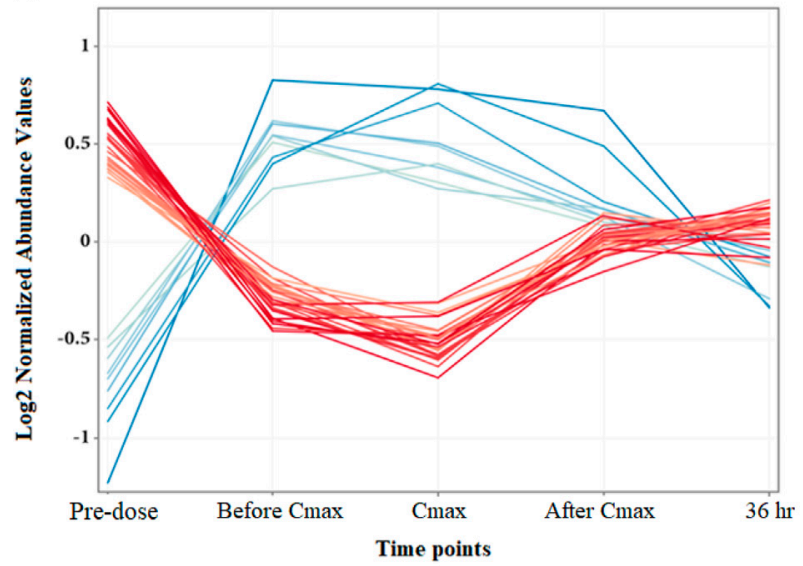

C

Eicosapentaenoic acid

12-HETE

4-HDoHE

11-Delydro-thromboxane $B 2$

12,13-EрOME

2-Hydroxydesogestrel

LysoPA(20:3(8Z,112,14Z)/0;0)

LysoPE(P-18:0/0:0)

Progesterone

21-Hydroxypregnenolone

Hexadecanedioic acid mono-L-carnitine

8,9-DiHETrE

Linoelaidic acid

PGF2a ethanolamide

17.Hydroxymethylethisterone

LysoPC(P-18:0/0:0)

Resohin D1

LysoPC(P-18:1(9Z):0:0)

13-L-Hydroperoxylinoleic acid

13-HOTE

12-KETE

Delnydroabietic acid

LysoPE(P-16:0/0:0)

Prostaglandin $\mathrm{H} 1$

14,15-DiHETE

Succinic acid

2-beta-hydroxymedroxyprogesterone

$\mathrm{N}$-Oleoylethanolamine

1,1-Dimethylbiguanide

N-Mononitrosopiperazine

2-methyl-1,3-thiazolidine-2-carboxamide

5-Hydroxymethyluracil

5-Aminopentanoic acid

4-Ethylphemylsulfate

Propionic acid

N-Lactoylphemylalanine

3-Hydroxyvalproic acid
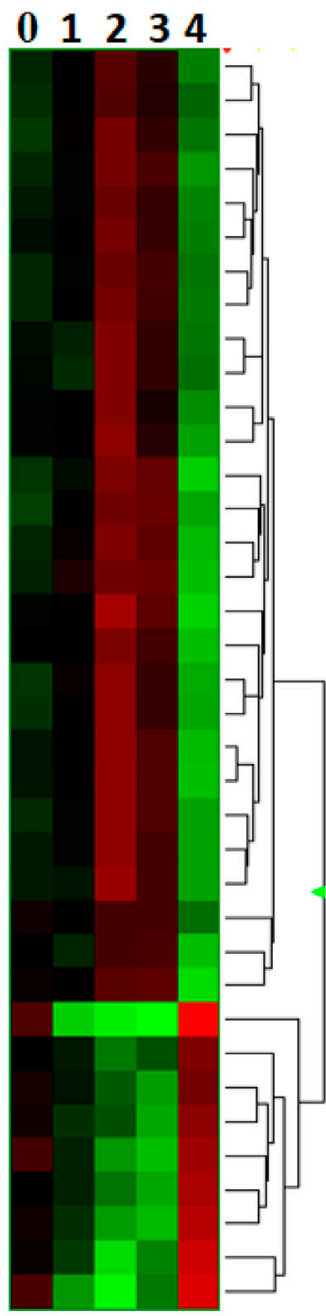

Color range

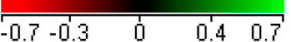

FIGURE 4 | Metformin-dependent metabolites. (A) Venn diagram showing significantly altered metabolites and metabolites with similar or opposite change in their metabolic levels compared to metformin levels at the five time points based on Pearson similarity test (R0.95-1). (B) The 28 (red line) and 8 (blue line) metformindependent metabolites showing opposite and similar trend in their levels to metformin, respectively. (C) Hierarchal clustering (HAC) and heatmap analysis of significantly altered metformin dependent metabolites. Time points $0,1,2,3$, and 4 refer to baseline, before $\mathrm{C}_{\max }, \mathrm{C}_{\max }$, after $\mathrm{C}_{\max }$ and $36 \mathrm{~h}$ post metformin administration. Metformin is 1,1-Dimethylbiguanide.

circulating levels) is associated with a range of conditions such as T2DM, obesity, cancer, and cardiovascular diseases (Nie et al., 2018; Siddik and Shin, 2019). The physiological roles of BCAA are mainly mediated via phosphoinositide 3-kinase/protein kinase $\mathrm{B} /$ mammalian target of rapamycin (PI3K/AKT/mTOR) signal pathway (Nie et al., 2018). Our findings indicate that one reason behind the multiple beneficial effects of metformin might be due to its significant impact on BCAA pathway. This is a plausible explanation since metformin has previously been reported to suppress BCAA catabolic enzyme expression or activity (Rivera et al., 2020) and PI3K/AKT/mTOR signal pathway (Zhao et al., 2018; Wang et al., 2019), which thereby will affect several crucial biochemical pathways.
Out of the 111 metabolites significantly altered by metformin administration, 36 metabolites displayed a change in their level similar or opposed to the metformin level pattern at the five-time points (Pearson similarity test $(\mathrm{R}=0.95-1)$. Therefore, these metabolites were considered as metformin-dependent metabolites. Amongst the metabolites that had a similar pattern to metformin is 5-hydroxymethyl uracil (5-hmU). 5$\mathrm{hmU}$ is one of the most enigmatic oxidative modifications of DNA, mainly formed by the oxidation/hydroxylation of thymine or ROS reaction with 5-methylcytosine (Olinski et al., 2016). It is a common oxidative DNA lesion; however, specific repair activities mainly through hmU-DNA glycosylase remove this modified base from DNA to limit mutagenesis, cytostasis, and 


\section{A}

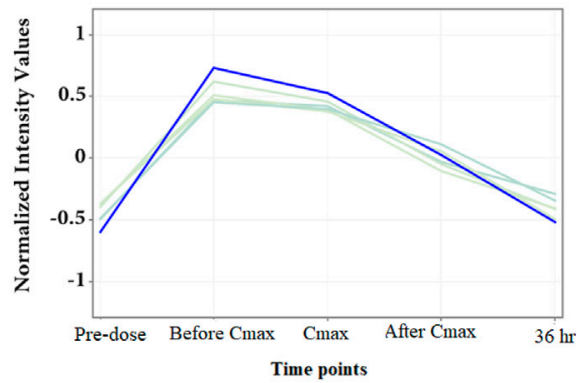

B

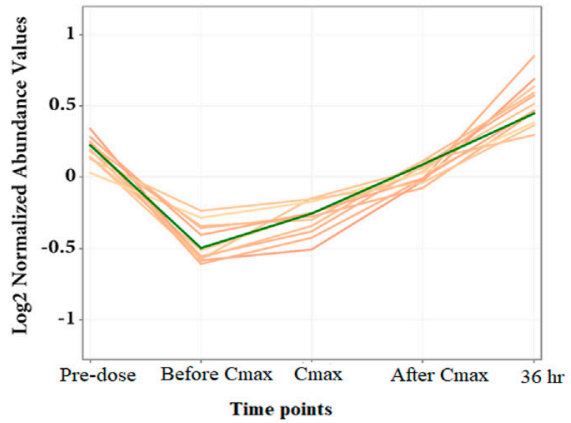

C

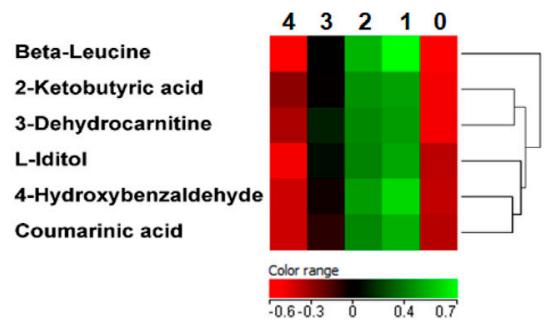

D

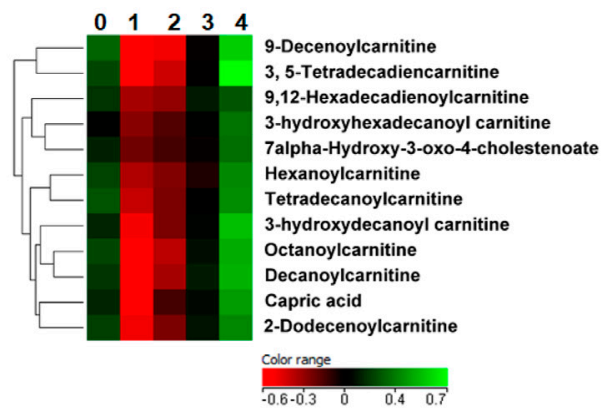

FIGURE 5 | The level of metformin-independent metabolites at the five-time points. (A, B) The 6 and 12 metformin-independent metabolites, respectively, initially increased or decreased, followed by a subsequent linear decrease or increase in their metabolic levels. (C, D) Hierarchal clustering (HAC) and heatmap analysis of significantly altered metformin independent metabolites. Time points $0,1,2,3$, and 4 refer to baseline, before $\mathrm{C}_{\max }, \mathrm{C}_{\max }$, after $\mathrm{C}_{\max }$, and $36 \mathrm{~h}$ post metformin administration.

cytotoxicity (Cooke et al., 2003). Thus, oxidative DNA damage has been implicated in cancer and aging. Our data suggest that metformin can alter the DNA lesion repair mechanisms, explaining its association with reduced cancers (Olinski et al., 2016). Interestingly, we have previously reported a dysregulation in the level of 5-hmU in metformin-treated diabetic patients, highlighting the importance of this metabolite in the underlying mechanisms of metformin (Aleidi et al., 2021).

There is growing evidence that abnormalities in the microbiota composition may contribute to the development of non-communicable diseases, including diabetes and obesity (Lazar et al., 2019). The composition of the gut microbiota (diversity or the abundance of particular species) is defined by a combination of factors including gender, age, body mass index, host genetics and immunity, and therapeutics drugs (Maurice et al., 2013; Zhernakova et al., 2016). It has been reported that some actions of metformin may be mediated by altering the gut microbial diversity and enriching beneficial bacteria (Tong et al., 2018; Arneth et al., 2019). In the current work, the levels of two microbial metabolites, ethylphenyl sulfate, and propionic acid, changed similarly to the metformin pattern. Propionic acid is a short-chain fatty acid mainly produced by the fermentation of undigested food by the colonic microbiota. Propionic acid inhibits lipolysis and induces lipogenesis in adipose tissue, suppresses fatty acid production in the liver, inhibits food intake, increases the duration of satiety, and exerts immunosuppressive actions (Al-Lahham et al., 2010; Lazar et al., 2019). These beneficial effects are associated with improved insulin sensitivity and reduced body weight (AlLahham et al., 2010). Unlike propionic acid, the function of ethylphenyl sulfate in the host physiology and pathophysiology is not yet elucidated (Lazar et al., 2019). The association between propionic acid and metformin levels detected in the current study suggests that metformin might acutely boost the capability of the gut bacteria to produce certain types of short-chain fatty acids such as propionic acid, which intern can suppress the appetite and alter blood glucose levels in different ways.

Dysregulation in lipid metabolism and increased lipogenesis have been recognized as a hallmark of cancer and linked with PCOS disorder (Menendez and Lupu, 2007). Metformindependent metabolites acutely changed in an opposed manner to metformin patterns were mainly lipids and lipid-like molecules, including polyunsaturated fatty acids (PUFA), eicosanoids, and glycerophospholipid (Figure 4). Several studies have shown that enzymes involved in the glycerophospholipid pathway may be used as potential targets for antitumor therapy (Dolce et al., 2011). In highly proliferating cancer cells, de novo fatty acids synthesis continually provides glycerophospholipids essential for membrane production (Kuhajda et al., 1994). The fact that metformin significantly decreased the level of several glycerophospholipids herein suggests that one mechanism by which metformin inhibits cancer cell growth could be by altering key enzymes involved in glycerophospholipid synthesis. Moreover, in line with our data, the downregulation effect of metformin on glycerophospholipids has 
been reported in women with PCOS. The latter might contribute to the reported favorable effect of metformin on dyslipidemia in PCOS (Pradas et al., 2019).

Eicosanoids, including prostaglandins (PG), leukotrienes, and hydroxyeicosatetraenoic acids (HETEs), are generated from the metabolism of arachidonic acid by cyclooxygenase (COX), lipoxygenase (LOX), and cytochrome 450 pathways (Wang and Dubois, 2010). Eicosanoids and related bioactive lipid mediators derived from PUFA are complex and challenging lipid network signaling. Eicosanoids are much appreciated for their pleiotropic effects and implication in various pathological conditions, including inflammation and cancer (Wang and Dubois, 2010; Dennis and Norris, 2015). Many HETE metabolites are wellknown pro-inflammatory eicosanoids that increase inflammatory cytokine expression inducing chronic inflammation associated with insulin resistance, metabolic syndrome, and T2DM (Chakrabarti et al., 2009; Guadarrama-Lopez et al., 2014). Additionally, proinflammatory eicosanoids, including PG and leukotrienes, can modulate tumor proliferation, apoptosis, migration, and invasion through multiple signaling pathways, and can remodel the tumor microenvironment with enhanced tumour angiogenesis (Wang and Dubois, 2010). In the current work, the levels of several metformindependent metabolites involved in the eicosanoid synthesis pathways, such as several HETEs and PGH involved in LOX and COX pathways, respectively, were decreased upon metformin administration and reserve to baseline level at 36-h post-dose. The presented findings indicate that the promising roles of metformin in improving insulin resistance, preventing cancer, and suppressing tumor progression might involve key enzymes in arachidonic acid metabolism particularly COX and LOX pathways. Our results are in line with (Hyun et al., 2013), who reported that metformin suppressed the protein level of COX-2 and may attenuate inflammatory responses. Moreover, recent in vitro and in vivo studies showed that a combination treatment of celecoxib (selective COX-2 inhibitor) and metformin inhibited the proliferation of Hepatocellular carcinoma to a greater extent than either treatment alone ( $\mathrm{Hu}$ et al., 2020). However, there is little known about metformin's effects on lipid and arachidonic acid metabolisms, and more in-depth studies to investigate its impact on these pathways remain urgent.

Several acylcarnitines showed an increase in their level that was independent of metformin level, Figure 5C. The level of these metabolites was initially decreased upon metformin administration and then linearly increased to return to normal level at $36 \mathrm{~h}$ post metformin administration. This suggests that the change in acylcarnitines level might be context-dependent and affected by factors other than metformin level, such as the pathological state of the subject.

Acylcarnitines are derived from L-carnitine esterification by carnitine acyltransferases during the $\beta$-oxidation of fatty acids in the mitochondria (Houten and Wanders, 2010). Several studies have demonstrated the importance of L-carnitine and acylcarnitines in fatty acid oxidation and the modulation of intracellular coenzyme A (CoA) homeostasis, maintaining normal mitochondrial functions (Reuter and Evans, 2012). These functions crucially influence physiological processes, including energy homeostasis, regulation of insulin secretion, and insulin sensitivity (Mai et al., 2013). Disturbances in the endogenous carnitine pool (L-carnitine and short, medium, and long-chain acylcarnitines) serve as a diagnostic marker for the equilibrium between acyl-CoA and acylcarnitine species. They can reflect mitochondrial and metabolic dysfunction (Reuter and Evans, 2012). Increased acylcarnitine levels have been proposed as markers of insulin resistance, T2DM and cardiovascular diseases (Koves et al., 2008). Our findings indicate that metformin might initially affect fatty acid $\beta$-oxidation by altering carnitine palmitoyltransferase activity and decreasing acylcarnitines' levels.

Coumarinic acid is another metformin-independent metabolite that increased upon metformin administration and then decreased to a normal level at $36 \mathrm{~h}$ post-dose. Coumarinic acid is a hydroxycinnamic acid derivative and one of the three isomers of coumaric acid: o-coumaric acid, m-coumaric acid, and p-coumaric acid. This class of phenolic compounds has been reported to possess potent antioxidant and anti-inflammatory properties both in vitro and in vivo by decreasing the expression of inflammatory mediator TNF- $\alpha$ and circulating immune complexes (Pragasam et al., 2013; Alam et al., 2016). Additionally, coumaric acids have been reported to exhibit marked antidiabetic action (Omar et al., 2016; Abdel-Moneim et al., 2018). o-coumaric acid or coumarinic acid was found to restore glycemic control and insulin sensitivity in rats fed high fats/high sucrose diet and suffered from hyperglycemia and insulin resistance (Omar et al., 2016).

Numerous favorable effects have been reported for metformin. Several lines of evidence implicate that metformin possesses hepatoprotective effect from injuries induced by hepatotoxic substances or liver conditions (Iranshahy et al., 2019). Alkaline phosphatase (ALP) is a well-known biomarker for liver disease, and its elevated blood level has been linked with liver injury (Saravi et al., 2016). In our work, metformin was associated with a significant decrease in the level of ALP, while no significant effect was detected on aminotransferase levels GOT and GPT (Table 1). This finding can be linked with the previously reported hepatoprotective effect of metformin, where metformin was found to normalize or decrease the levels of ALP enzyme (Saravi et al., 2016). Noteworthy, metabolomics data identified one subject with different metabolic profiles, even before metformin administration, that had a significant increase in the liver enzymes after metformin intake. This finding points to the fact that individuals may vary in response to this therapy according to their metabolic pattern and highlights the important role of metabolomics in personalized medicine.

Of note, the identified metabolites linked to metformin intake herein were dysregulated after a single dose of metformin. Therefore, a follow-up study in the future under chronic conditions remains essential to evaluate and validate their link to metformin pharmacodynamics, and their role as potential biomarkers to monitor the pharmacological effect of metformin.

\section{CONCLUSION}

In the present study, MS-based untargeted metabolic profiling was applied for the first time to uncover the biochemical 
changes induced by metformin at different time points in healthy subjects.

Our findings revealed that BCAA pathway was the most significantly altered pathway by metformin. Additionally, specific metabolites that showed metformin-dependent changes in their levels were identified, including 5-hmU, propionic acid, and several eicosanoids. The altered metformin-dependent metabolites pointed to fundamental biochemical processes by which metformin can exert its multiple beneficial effects, including lipid network signaling, inflammation, energy homeostasis, DNA lesion repair mechanisms, and gut microbiota functions. Thus, the distinctive metabolic pattern linked to metformin intake, particularly metformin-dependent metabolites, can be used as potential biomarkers or metabolic signature to predict the potential effect and mechanism of actions of new chemical entities during drug development.

Further studies with larger sample sizes and under chronic conditions are necessary to evaluate the link between the dysregulated metabolites and metformin pharmacodynamics and investigate their role as potential biomarkers to monitor the pharmacological effect of metformin. Once validated, metformin-dependent endogenous metabolites might be utilized in conjunction with other existing biomarkers to monitor metformin's efficacy for personalized medicine, especially since metformin is not metabolized inside the body and is excreted unchanged urine.

Future research is warranted to investigate the effect of metformin on BCAA pathway and, most importantly, to identify the endogenous lipid pattern induced by metformin. Refined lipidomics methodologies can be applied to provide a deeper understanding of the role of metformin in arachidonic acids metabolism and eicosanoids signaling and identify new potential targets for inflammatory and metabolic conditions.

\section{DATA AVAILABILITY STATEMENT}

The raw data supporting the conclusions of this article will be made available by the authors, without undue reservation, to any qualified researcher.

\section{REFERENCES}

Abdel-Moneim, A., El-Twab, S. M. A., Yousef, A. I., Reheim, E. S. A., and Ashour, M. B. (2018). Modulation of Hyperglycemia and Dyslipidemia in Experimental Type 2 Diabetes by Gallic Acid and P-Coumaric Acid: The Role of Adipocytokines and PPAR $\gamma$. Biomed. Pharmacother. 105, 1091-1097. doi:10.1016/j.biopha.2018.06.096

Adam, J., Brandmaier, S., Leonhardt, J., Scheerer, M. F., Mohney, R. P., Xu, T., et al. (2016). Metformin Effect on Nontargeted Metabolite Profiles in Patients with Type 2 Diabetes and in Multiple Murine Tissues. Diabetes 65, 3776-3785. doi: $10.2337 / \mathrm{db} 16-0512$

Alam, M. A., Subhan, N., Hossain, H., Hossain, M., Reza, H. M., Rahman, M. M., et al. (2016). Hydroxycinnamic Acid Derivatives: a Potential Class of Natural Compounds for the Management of Lipid Metabolism and Obesity. Nutr. Metab. 13, 27. doi:10.1186/s12986-016-0080-3

\section{ETHICS STATEMENT}

The studies involving human participants were reviewed and approved by Institutional Review Board at Jordan Center for Pharmaceutical Research at Amman, Jordan (IRB-01-R02) and Institutional Review Board (IRB) at King Saud University (approval number E-19-4234). The patients/participants provided their written informed consent to participate in this study.

\section{AUTHOR CONTRIBUTIONS}

$\mathrm{LD}$ and $\mathrm{AR}$, designed the project, supervised the experiment, analyzed data, and drafted the manuscript. TA supervised and executed the clinical study. HB, MM, AA data collection and methodology. All the authors revised and approved the final version of the manuscript.

\section{FUNDING}

This research project was supported by the University Diabetes Center, King Saud University Medical City, King Saud University.

\section{ACKNOWLEDGMENTS}

The authors would like to express their gratitude to the Executive Director of Center for Genome Medicine and Dr. Brian Meyer, Chairman of the Department of Clinical Genomics, King Faisal Specialist Hospital and Research Centre, for the support and all the subjects. Also the authors would like to expand thanks to king Saud University Medical City for its support.

\section{SUPPLEMENTARY MATERIAL}

The Supplementary Material for this article can be found online at: https://www.frontiersin.org/articles/10.3389/fphar.2021.705932/ full\#supplementary-material

Aleidi, S. M., Dahabiyeh, L. A., Gu, X., Al Dubayee, M., Alshahrani, A., Benabdelkamel, H., et al. (2021). Obesity Connected Metabolic Changes in Type 2 Diabetic Patients Treated with Metformin. Front. Pharmacol. 11, 616157. doi:10.3389/fphar.2020.616157

Al-Lahham, S. a. H., Peppelenbosch, M. P., Roelofsen, H., Vonk, R. J., and Venema, K. (2010). Biological Effects of Propionic Acid in Humans; Metabolism, Potential Applications and Underlying Mechanisms. Biochim. Biophys. Acta (Bba) - Mol. Cel Biol. Lipids 1801, 1175-1183. doi:10.1016/j.bbalip.2010.07.007

Arneth, B., Arneth, R., and Shams, M. (2019). Metabolomics of Type 1 and Type 2 Diabetes. Int. J. Mol. Sci. 20, 2467. doi:10.3390/ijms20102467

Balashova, E. E., Maslov, D. L., and Lokhov, P. G. (2018). A Metabolomics Approach to Pharmacotherapy Personalization. J. Personalized Med. 8, 28. doi:10.3390/jpm8030028

Bao, Y., Zhao, T., Wang, X., Qiu, Y., Su, M., Jia, W., et al. (2009). Metabonomic Variations in the Drug-Treated Type 2 Diabetes Mellitus Patients and Healthy Volunteers. J. Proteome Res. 8, 1623-1630. doi:10.1021/pr800643w 
Bonnefont-Rousselot, D., Raji, B., Walrand, S., Gardès-Albert, M., Jore, D., Legrand, A., et al. (2003). An Intracellular Modulation of Free Radical Production Could Contribute to the Beneficial Effects of Metformin towards Oxidative Stress. Metabolism 52, 586-589. doi:10.1053/meta.2003.50093

Buse, J. B., Wexler, D. J., Tsapas, A., Rossing, P., Mingrone, G., Mathieu, C., et al. (2020). 2019 Update to: Management of Hyperglycemia in Type 2 Diabetes, 2018. A Consensus Report by the American Diabetes Association (ADA) and the European Association for the Study of Diabetes (EASD). Dia Care 43, 487-493. doi:10.2337/dci19-0066

Cameron, A. R., Morrison, V. L., Levin, D., Mohan, M., Forteath, C., Beall, C., et al. (2016). Anti-Inflammatory Effects of Metformin Irrespective of Diabetes Status. Circ. Res. 119, 652-665. doi:10.1161/circresaha.116.308445

Chakrabarti, S. K., Cole, B. K., Wen, Y., Keller, S. R., and Nadler, J. L. (2009). 12/15Lipoxygenase Products Induce Inflammation and Impair Insulin Signaling in 3T3-L1 Adipocytes. Obesity 17, 1657-1663. doi:10.1038/oby.2009.192

Chong, J., Soufan, O., Li, C., Caraus, I., Li, S., Bourque, G., et al. (2018). MetaboAnalyst 4.0: towards More Transparent and Integrative Metabolomics Analysis. Nucleic Acids Res. 46, W486-W494. doi:10.1093/ nar/gky310

Cooke, M. S., Evans, M. D., Dizdaroglu, M., and Lunec, J. (2003). Oxidative DNA Damage: Mechanisms, Mutation, and Disease. FASEB j. 17, 1195-1214. doi:10.1096/fi.02-0752rev

Dahabiyeh, L. A., Malkawi, A. K., Wang, X. H., Colak, D., Mujamammi, A. H., Sabi, E. M., et al. (2020). Dexamethasone-Induced Perturbations in Tissue Metabolomics Revealed by Chemical Isotope Labeling LC-MS Analysis. Metabolites 10, 42. doi:10.3390/metabo10020042

Dahabiyeh, L. A., Mahmoud, N. N., Al-Natour, M. A., Safo, L., Kim, D. H., Khalil, E. A., et al. (2021). Phospholipid-Gold Nanorods Induce Energy Crisis in MCF7 Cells: Cytotoxicity Evaluation Using LC-MS-Based Metabolomics Approach. Biomolecules 11, 364. doi:10.3390/biom11030364

Davies, M. J., D’Alessio, D. A., Fradkin, J., Kernan, W. N., Mathieu, C., Mingrone, G., et al. (2018). Management of Hyperglycemia in Type 2 Diabetes, 2018. A Consensus Report by the American Diabetes Association (ADA) and the European Association for the Study of Diabetes (EASD). Dia Care 41, 2669-2701. doi:10.2337/dci18-0033

Dennis, E. A., and Norris, P. C. (2015). Eicosanoid Storm in Infection and Inflammation. Nat. Rev. Immunol. 15, 511-523. doi:10.1038/nri3859

Dolce, V., Cappello, A. R., Lappano, R., and Maggiolini, M. (2011). Glycerophospholipid Synthesis as a Novel Drug Target against Cancer. Curr. Mol. Pharmacol. 4, 167-75. doi:10.2174/1874467211104030167

Foretz, M., Guigas, B., Bertrand, L., Pollak, M., and Viollet, B. (2014). Metformin: From Mechanisms of Action to Therapies. Cel Metab. 20, 953-966. doi:10.1016/ j.cmet.2014.09.018

Foretz, M., Guigas, B., and Viollet, B. (2019). Understanding the Glucoregulatory Mechanisms of Metformin in Type 2 Diabetes Mellitus. Nat. Rev. Endocrinol. 15, 569-589. doi:10.1038/s41574-019-0242-2

Gu, X. Y., Al Dubayee, M., Alshahrani, A., Masood, A., Benabdelkamel, H., Zahra, M., et al. (2020). Distinctive Metabolomics Patterns Associated with Insulin Resistance and Type 2 Diabetes Mellitus. Front. Mol. Biosci. 7, 609806. doi:10.3389/fmolb.2020.609806

Guadarrama-Lopez, A. L., Valdes-Ramos, R., and Martinez-Carrillo, B. E. (2014). Type 2 Diabetes, PUFAs, and Vitamin D: Their Relation to Inflammation. J. Immunol. Res. 2014, 860703. doi:10.1155/2014/860703

Guasch-Ferré, M., Hruby, A., Toledo, E., Clish, C. B., Martínez-González, M. A., Salas-Salvadó, J., et al. (2016). Metabolomics in Prediabetes and Diabetes: A Systematic Review and Meta-Analysis. Dia Care 39, 833-846. doi:10.2337/ dc15-2251

Houten, S. M., and Wanders, R. J. A. (2010). A General Introduction to the Biochemistry of Mitochondrial Fatty Acid $\beta$-oxidation. J. Inherit. Metab. Dis. 33, 469-477. doi:10.1007/s10545-010-9061-2

Hu, J.-W., Chen, B., Zhang, J., Qi, Y.-P., Liang, J.-H., Zhong, J.-H., et al. (2020). Novel Combination of Celecoxib and Metformin Improves the Antitumor Effect by Inhibiting the Growth of Hepatocellular Carcinoma. J. Cancer 11, 6437-6444. doi:10.7150/jca.47532

Hyun, B., Shin, S., Lee, A., Lee, S., Song, Y., and Ha, N.-J. (2013). Metformin DownRegulates TNF- $\alpha$ Secretion via Suppression of Scavenger Receptors in Macrophages. Immune Netw. 13, 123-132. doi:10.4110/in.2013.13.4.123
Iranshahy, M., Rezaee, R., and Karimi, G. (2019). Hepatoprotective Activity of Metformin: A New mission for an Old Drug?. Eur. J. Pharmacol. 850, 1-7. doi:10.1016/j.ejphar.2019.02.004

Jacob, M., Lopata, A. L., Dasouki, M., and Abdel Rahman, A. M. (2019). Metabolomics toward Personalized Medicine. Mass. Spec. Rev. 38, 221-238. doi:10.1002/mas.21548

Kane, D. A., Anderson, E. J., Price III, J. W., Woodlief, T. L., Lin, C.-T., Bikman, B. T., et al. (2010). Metformin Selectively Attenuates Mitochondrial H2O2 Emission without Affecting Respiratory Capacity in Skeletal Muscle of Obese Rats. Free Radic. Biol. Med. 49, 1082-1087. doi:10.1016/ j.freeradbiomed.2010.06.022

Koves, T. R., Ussher, J. R., Noland, R. C., Slentz, D., Mosedale, M., Ilkayeva, O., et al. (2008). Mitochondrial Overload and Incomplete Fatty Acid Oxidation Contribute to Skeletal Muscle Insulin Resistance. Cel Metab. 7, 45-56. doi:10.1016/j.cmet.2007.10.013

Kuhajda, F. P., Jenner, K., Wood, F. D., Hennigar, R. A., Jacobs, L. B., Dick, J. D., et al. (1994). Fatty Acid Synthesis: a Potential Selective Target for Antineoplastic Therapy. Proc. Natl. Acad. Sci. 91, 6379-6383. doi:10.1073/ pnas.91.14.6379

Lazar, V., Ditu, L. M., Pircalabioru, G. G., Picu, A., Petcu, L., Cucu, N., et al. (2019). Gut Microbiota, Host Organism, and Diet Trialogue in Diabetes and Obesity. Front. Nutr. 6, 21. doi:10.3389/fnut.2019.00021

Liu, X., Romero, I. L., Litchfield, L. M., Lengyel, E., and Locasale, J. W. (2016). Metformin Targets Central Carbon Metabolism and Reveals Mitochondrial Requirements in Human Cancers. Cel Metab. 24, 728-739. doi:10.1016/ j.cmet.2016.09.005

Ma, X., Han, M., Li, D., Hu, S., Gilbreath, K. R., Bazer, F. W., et al. (2017). L-Arginine Promotes Protein Synthesis and Cell Growth in Brown Adipocyte Precursor Cells via the mTOR Signal Pathway. Amino Acids 49, 957-964. doi:10.1007/s00726-017-2399-0

Mai, M., Tonjes, A., Kovacs, P., Stumvoll, M., Fiedler, G. M., and Leichtle, A. B. (2013). Serum Levels of Acylcarnitines Are Altered in Prediabetic Conditions. Plos One 8, e82459. doi:10.1371/journal.pone.0082459

Malkawi, A. K., Alzoubi, K. H., Jacob, M., Matic, G., Ali, A., Al Faraj, A., et al. (2018). Metabolomics Based Profiling of Dexamethasone Side Effects in Rats. Front. Pharmacol. 9, 46. doi:10.3389/fphar.2018.00046

Maurice, C. F., Haiser, H. J., and Turnbaugh, P. J. (2013). Xenobiotics Shape the Physiology and Gene Expression of the Active Human Gut Microbiome. Cell 152, 39-50. doi:10.1016/j.cell.2012.10.052

Menendez, J. A., and Lupu, R. (2007). Fatty Acid Synthase and the Lipogenic Phenotype in Cancer Pathogenesis. Nat. Rev. Cancer 7, 763-777. doi:10.1038/ $\operatorname{nrc} 2222$

Nie, C. X., He, T., Zhang, W. J., Zhang, G. L., and Ma, X. (2018). Branched Chain Amino Acids: Beyond Nutrition Metabolism. Int. J. Mol. Sci. 19. doi:10.3390/ ijms19040954

Olinski, R., Starczak, M., and Gackowski, D. (2016). Enigmatic 5hydroxymethyluracil: Oxidatively Modified Base, Epigenetic Mark or Both? Mutat. Res. Rev. Mutat. Res. 767, 59-66. doi:10.1016/j.mrrev.2016.02.001

Omar, H. E.-D. M., Ragaa, S. M., Abd Elghaffar, S. K., Alduraywish, A. A., and ElMetwally, T. H. (2016). Berberine, Quercetin and O-Coumaric Acid Phytochemicals Ameliorate the Impact of ExperimentallyFed High-Fat/ High-Sucrose Diet on Pancreas B-Cells and Glycemic Control Indices. Austin J. Endocrinol. Diabetes 3, 1042

Pradas, I., Rovira-Llopis, S., Naudi, A., Banuls, C., Rocha, M., Hernandez-Mijares, A., et al. (2019). Metformin Induces Lipid Changes on Sphingolipid Species and Oxidized Lipids in Polycystic Ovary Syndrome Women. Scientific Rep. 9, 16033. doi:10.1038/s41598-019-52263-w

Pragasam, S. J., Venkatesan, V., and Rasool, M. (2013). Immunomodulatory and Anti-inflammatory Effect of P-Coumaric Acid, a Common Dietary Polyphenol on Experimental Inflammation in Rats. Inflammation 36, 169-176. doi:10.1007/s10753-012-9532-8

Rena, G., Hardie, D. G., and Pearson, E. R. (2017). The Mechanisms of Action of Metformin. Diabetologia 60, 1577-1585. doi:10.1007/s00125-017-4342-z

Reuter, S. E., and Evans, A. M. (2012). Carnitine and Acylcarnitines. Clin. Pharmacokinet. 51, 553-572. doi:10.1007/bf03261931

Rivera, M. E., Lyon, E. S., and Vaughan, R. A. (2020). Effect of Metformin on Myotube BCAA Catabolism. J. Cel Biochem 121, 816-827. doi:10.1002/jcb.29327 
Rotermund, C., Machetanz, G., and Fitzgerald, J. C. (2018). The Therapeutic Potential of Metformin in Neurodegenerative Diseases. Front. Endocrinol. 9, 400. doi:10.3389/fendo.2018.00400

Ryan, P. M., Patterson, E., Carafa, I., Mandal, R., Wishart, D. S., Dinan, T. G., et al. (2020). Metformin and Dipeptidase Peptidyl-4 Inhibitor Differentially Modulate the Intestinal Microbiota and Plasma Metabolome of Metabolically Dysfunctional Mice. Can. J. Diabetes. 44, 146-155. doi:10.1016/j.jcjd.2019.05.008

Sanchez-Rangel, E., and Inzucchi, S. E. (2017). Metformin: Clinical Use in Type 2 Diabetes. Diabetologia 60, 1586-1593. doi:10.1007/s00125-017-4336-x

Saeedi Saravi, S. S., Hasanvand, A., Shahkarami, K., and Dehpour, A. R. (2016). The Protective Potential of Metformin against Acetaminophen-Induced Hepatotoxicity in BALB/C Mice. Pharm. Biol. 54, 2830-2837. doi:10.1080/ 13880209.2016.1185633

Seifarth, C., Schehler, B., and Schneider, H. J. (2013). Effectiveness of Metformin on Weight Loss in Non-diabetic Individuals with Obesity. Exp. Clin. Endocrinol. Diabetes 121, 27-31. doi:10.1055/s-0032-1327734

Siddik, M. A. B., and Shin, A. C. (2019). Recent Progress on Branched-Chain Amino Acids in Obesity, Diabetes, and beyond. Endocrinol. Metab. 34, 234-246. doi:10.3803/enm.2019.34.3.234

Song, R. (2016). Mechanism of Metformin: A Tale of Two Sites. Dia Care 39, 187-189. doi:10.2337/dci15-0013

Tong, X. L., Xu, J., Lian, F. M., Yu, X. T., Zhao, Y. F., Xu, L. P., et al. (2018). Structural Alteration of Gut Microbiota during the Amelioration of Human Type 2 Diabetes with Hyperlipidemia by Metformin and a Traditional Chinese Herbal Formula: a Multicenter, Randomized, Open Label Clinical Trial. Mbio 9, e02392-17. doi:10.1128/mbio.02392-17

Vinaixa, M., Rodriguez, M. A., Samino, S., Diaz, M., Beltran, A., Mallol, R., et al. (2011). Metabolomics Reveals Reduction of Metabolic Oxidation in Women with Polycystic Ovary Syndrome after Pioglitazone-Flutamide-Metformin Polytherapy. Plos One 6, e29052. doi:10.1371/journal.pone.0029052

Viollet, B., Guigas, B., Garcia, N. S., Leclerc, J., Foretz, M., and Andreelli, F. (2012). Cellular and Molecular Mechanisms of Metformin: an Overview. Clin. Sci. (Lond) 122, 253-270. doi:10.1042/cs20110386
Wang, D., and Dubois, R. N. (2010). Eicosanoids and Cancer. Nat. Rev. Cancer 10, 181-193. doi: $10.1038 / \mathrm{nrc} 2809$

Wang, Z., Guo, J. J., Han, X. Q., Xue, M., Wang, W. M., Mi, L., et al. (2019). Metformin Represses the Pathophysiology of AAA by Suppressing the Activation of PI3K/AKT/mTOR/autophagy Pathway in ApoE(-/-) Mice. Cel Biosci. 9, 68. doi:10.1186/s13578-019-0332-9

Worley, B., and Powers, R. (2013). Multivariate Analysis in Metabolomics. Curr. Metabolomics 1, 92-107. doi:10.2174/2213235X11301010092

Zhao, Y., Sun, H., Feng, M., Zhao, J., Zhao, X., Wan, Q., et al. (2018). Metformin Is Associated with Reduced Cell Proliferation in Human Endometrial Cancer by Inbibiting PI3K/AKT/mTOR Signaling. Gynecol. Endocrinol. 34, 428-432. doi:10.1080/09513590.2017.1409714

Zhernakova, A., Kurilshikov, A., Bonder, M. J., Tigchelaar, E. F., Schirmer, M., Vatanen, T., et al. (2016). Population-based Metagenomics Analysis Reveals Markers for Gut Microbiome Composition and Diversity. Science 352, 565-569. doi:10.1126/science.aad3369

Zhu, Y., Feng, Y., Shen, L., Xu, D., Wang, B., Ruan, K., et al. (2013). Effect of Metformin on the Urinary Metabolites of Diet-Induced-Obese Mice Studied by Ultra Performance Liquid Chromatography Coupled to Time-Of-Flight Mass Spectrometry (UPLC-TOF/MS). J. Chromatogr. B 925, 110-116. doi:10.1016/ j.jchromb.2013.02.040

Conflict of Interest: The authors declare that the research was conducted in the absence of any commercial or financial relationships that could be construed as a potential conflict of interest.

Copyright (c) 2021 Dahabiyeh, Mujammami, Arafat, Benabdelkamel, Alfadda and Abdel Rahman. This is an open-access article distributed under the terms of the Creative Commons Attribution License (CC BY). The use, distribution or reproduction in other forums is permitted, provided the original author(s) and the copyright owner(s) are credited and that the original publication in this journal is cited, in accordance with accepted academic practice. No use, distribution or reproduction is permitted which does not comply with these terms. 